\title{
M2 microglia promotes neurogenesis and oligodendrogenesis from neural stem/progenitor cells via the PPARY signaling pathway
}

\author{
Jichao Yuan ${ }^{1,2}$, Hongfei Ge ${ }^{1}$, Wei Liu ${ }^{1}$, Haitao Zhu ${ }^{1}$, Yaxing Chen ${ }^{1}$, Xuan Zhang $^{1}$, \\ Yang Yang ${ }^{1}$, Yi Yin ${ }^{1}$, Weixiang Chen ${ }^{1}$, Wanjiang Wu ${ }^{1}$, Yunfeng Yang ${ }^{1}$, Jiangkai Lin ${ }^{1}$ \\ ${ }^{1}$ Department of Neurosurgery and Key Laboratory of Neurotrauma, Southwest Hospital, Third Military Medical University, \\ Chongqing 400038, China \\ ${ }^{2}$ Department of Neurology, Southwest Hospital, Third Military Medical University, Chongqing 400038, China \\ Correspondence to: Jiangkai Lin, email: jklin@tmmu.edu.cn
}

Keywords: NSPCs, differentiation, M2 microglia, 15d-PGJ ${ }_{2}$ PPARY

Received: May 17, 2016

Accepted: January 23, 2017

Published: February 28, 2017

\section{ABSTRACT}

Neural stem/progenitor cells (NSPCs) are an important source of cells for cell replacement therapy after nerve injury. How to induce NSPCs differentiation towards neurons and oligodendrocytes is a challenging issue in neuroscience research. In the present study, we polarized microglia into M1 and M2 phenotype, used their supernatants to induce NSPCs differentiation, and investigated the effects of different microglia phenotypes on NSPCs differentiation and their mechanisms. We discovered that, after exposure to M1 phenotype supernatant, NSPCs differentiated into fewer Tuj-1+ and Olig2+ cells, but more GFAP+ cells. Meanwhile, a significantly increased number of Tuj-1+ and Olig2+ cells and smaller number of GFAP+ cells were generated by M2 microglia supernatant-induced NSPCs differentiation. We also observed that $15 \mathrm{~d}-\mathrm{PG} \mathrm{J}_{2}$, an endogenous ligand of PPARY, was elevated in M2 phenotype supernatant and could activate PPARY expression in NSPCs, whereas use of the PPARy inhibitor GW9662, could reduce the percentage of differentiated neurons and oligodendrocytes. Our study results confirm that M2 microglia supernatant can activate the PPARY signaling pathway and promote neurogenesis and oligodendrogenesis from NSPCs differentiation. The present study provides a further theoretical basis for induction of NSPCs oriented differentiation.

\section{INTRODUCTION}

Neural stem/progenitor cells (NSPCs) have a strong capacity for self-proliferation and differentiation. Thus they have great potential for use as replacement cells and play an important role in neural repair after central nervous system (CNS) injury [1]. However, due to the loss of various nutritional factors and the persistent secondary inflammation, more NSPCs are differentiated into astrocytes and ultimately form glial scars, which inhibit axon regeneration [2-4]. Therefore, it is of great importance to find an effective way to promote neurogenesis and oligodendrogenesis from NSPCs differentiation.

Inflammation is a key factor affecting NSPCs differentiation [5]. As resident immune cell in the CNS, microglia can be rapidly activated after injury, and participate in local inflammatory reaction [6]. Microglia can exert beneficial [7] or harmful $[8,9]$ effects, and this mainly depends on the two different microglia subtypes. Proinflammatory M1 microglia activated by tumor necrosis factor alpha (TNF-a) and interferon $\gamma(\mathrm{IFN}-\gamma)$ via classical pathways disrupts the internal environment. In contrast, anti-inflammatory M2 microglia activated by interleukin 4 (IL-4) and interleukin 13 (IL-13) via selective pathways plays a neuroprotective role $[10,11]$. It has been reported that microglia activated by IL-4 can promote neurogenesis and oligodendrogenesis from NSPCs differentiation [12], but the specific mechanism of action is unclear.

Prostaglandin $\mathrm{D}_{2}\left(\mathrm{PGD}_{2}\right)$, a type of cyclopentenone prostaglandin, is formed by the cyclooxygenation of arachidonic acid. 15-Deoxy- $\Delta$ 12,14-prostaglandin $\mathrm{J}_{2}$ 
$\left(15 \mathrm{~d}-\mathrm{PGJ}_{2}\right)$ is the ultimate metabolite of $\mathrm{PGD}_{2}$ through spontaneous nonenzymatic dehydration followed by isomerization and is an endogenous ligand for PPAR $\gamma$ [13]. 15D-PGJ ${ }_{2}$ possesses anti-inflammatory properties and plays a protective role in CNS injury [14-16]. It has been reported that anti-inflammatory macrophages could release $15 \mathrm{~d}_{-} \mathrm{PGJ}_{2}[17-19]$ and that $15 \mathrm{~d}-\mathrm{PGJ}_{2}$ could promote the proliferation of NSPCs via the PPAR $\gamma$ pathway [20]. Therefore, we speculated that $15 \mathrm{~d}_{-} \mathrm{PGJ}_{2}$ and PPAR $\gamma$ participate in M2 microglia-induced NSPCs differentiation.

In the present study, M1 and M2 microglia supernatants are used to induce NSPCs differentiation; and the differentiation into neurons, oligodendrocytes, and astrocytes is observed; the possible underlying mechanisms are also explored. This study provides an experimental evidence for improving the internal environment of the body after nerve injury and promoting NSPCs differentiation, and it furnishes certain interventional measures and targets for neural function recovery at later stages after CNS injury.

\section{RESULTS}

\section{Polarization of microglia}

The M0 microglia induced by serum-free culture medium were of irregular morphology; the expression levels of M1 subtype markers (iNOS and CD86) and M2 subtype markers (CD206 and Arg1) were all low, as the microglia had not yet been activated. After induction by LPS+IFN- $\gamma$ for 24 hours, the microglia became round and the number of microglia expressing iNOS and CD86 was significantly increased, whereas the expression levels of both CD206 and Arg1 were low. After induction by IL-4, the microglia exhibited long and spindle-like morphology, and the number of cells expressing CD206 and Arg1 was significantly increased, while the expression levels of iNOS and CD86 were relatively low (Figure 1). WB and PCR assays were consistent with these findings. These results demonstrated that we had successfully polarized microglia cultured in vitro into M1 and M2 subtypes.

To prove that microglia can maintain M1 and M2 phenotype without intervention, we measured the expression levels of M1 and M2 subtype markers after the intervening factors were removed (Figure 2). An immunofluorescence assay revealed that the levels of iNOS and CD86 expression in LPS + IFN- $\gamma$ induced cells were somewhat reduced compared to those before the intervention was removed, but approximately half of the cells continued to express M1 subtype markers. Additionally, approximately $50 \%$ of M2 microglia induced by IL-4 still expressed CD206 and Arg1, and the levels of mRNA were also elevated. These data indicated that the supernatants we collected contained high levels of M1 and M2 microglia secretions.

\section{NSPCs characteristics}

After three days of culture, NSPCs were globular in shape and grew in a suspended manner. Approximately $60 \%$ of cells expressed nestin and SOX2, which are the NSPCs-specific markers. After adherence to walls and differentiation for 14 days, the neurospheres expressed neuronal marker Tuj-1, oligodendrocyte marker Olig2, and astrocyte marker GFAP (Figure 3). The terminal differentiation markers (MAP2 and O4) were also consistent with these findings (Supplementary Figure 1). These results showed that the cells we cultured had improved differentiation potential and could be differentiated into various major cell subtypes in the central nervous system.

\section{M0, M1 and M2 microglia supernatants do not affect the apoptosis and proliferation of NSPCs}

With apoptosis assay and LDH release assay, we found that there were no statistically significant differences among M0, M1 and M2 microglia on day 3, 7 and 14 (Supplementary Figure 2). These data indicated that M0, M1 and M2 microglia had no different influence on the death/survival of NSPCs. Similarly, CCK-8 proliferation assay (Supplementary Figure 2) showed that different phenotypes had no different influence on the proliferation of NSPCs.

\section{M2 microglia supernatant promotes NSPCs differentiation towards neurons and oligodendrocytes}

Three days after NSPCs extraction, we added supernatants from polarized microglia to induce NSPCs adherence and differentiation (Figure 4). We found that on day 14 of differentiation, approximately $40 \%$ of NSPCs induced by M0 supernatant in the control group were differentiated into GFAP + astrocytes, with Tuj-1+ and Olig2+ cells each accounting for approximately $10 \%$. After induction by M1 supernatant, the percentages of Tuj-1+ and Olig2+ cells were further reduced, and more $\mathrm{GFAP}+$ differentiated cells were generated. In contrast, NSPCs induced by M2 supernatant were more likely to differentiate into Tuj-1+ cells (approximately 20\%), Olig2+ cells (approximately 30\%), and significantly decreased GFAP+ cells (only approximately 20\%). A WB assay showed that the expression levels of Tuj-1 and Olig2 were significantly elevated at day 7 and 14 after M2 microglia induction compared to M1 microglia supernatant induction, while the level of GFAP expression was significantly reduced. These results suggested that M2 microglia supernatant increased the numbers of neurons and oligodendrocytes generated by NSPCs differentiation, while reducing the differentiation ratio of astrocytes. 


\section{Higher level of 15d-PGJ2 in M2 microglia supernatant}

We further explored the amount of $15 \mathrm{~d}-\mathrm{PGJ} \mathrm{J}_{2}$ during microglia polarization, this compound has been identified as a natural ligand for PPAR $\gamma$ (Peroxisome proliferatoractivated receptor $\gamma$ ). LC-MS (Figure 5A-5E) and ELISA (Figure 5F) analyses detected ever little $15 \mathrm{~d}_{-} \mathrm{PGJ}_{2}$ in the M1 supernatant, whereas the amount of $15 \mathrm{~d}-\mathrm{PGJ}_{2}$ in the M2 supernatant was very abundant (Figure 5E, 5F).

\section{PPAR $\gamma$ participates in NSPCs differentiation induced by M2 microglia supernatant}

To investigate the mechanism by which $15 \mathrm{~d}-\mathrm{PGJ}_{2}$ from M2 microglia affects NSPCs differentiation, we studied PPAR $\gamma$ expression in NSPCs on day 14 (Figure 5G, 5H). We found that the expression of PPAR $\gamma$ in M2 supernatant-induced NSPCs was increased, whereas its expression in M0 and M1 supernatant-induced NSPCs was was low. These results were consistent with $15 \mathrm{~d}-\mathrm{PGJ}{ }_{2}$ released. $15 \mathrm{D}-\mathrm{PGJ}_{2}$ is an endogenous ligand of PPAR $\gamma$. Therefore, we speculated that $15 \mathrm{~d}-\mathrm{PGJ}_{2}$ activated PPAR $\gamma$ to promote NSPCs differentiation into neurons and oligodendrocytes.

\section{Inhibition of PPAR $\gamma$ reduces the percentage of neurons and oligodendrocytes generated by NSPCs differentiation}

To verify this hypothesis, we used a $15 \mathrm{~d}-\mathrm{PGJ}_{2}$ monomer and the PPAR $\gamma$ inhibitor GW9662 to intervene in NSPCs differentiation (Figure 6). We found that 15d-PGJ ${ }_{2}$ activated PPAR $\gamma$ expression and GW9662 suppressed PPAR $\gamma$ expression. There were no significant differences in Tuj-1, Olig2 or GFAP protein expression between the M2 group and the M1+15d-PGJ ${ }_{2}$ group. These data indicated that $15 \mathrm{~d}-\mathrm{PGJ}_{2}$ could promote

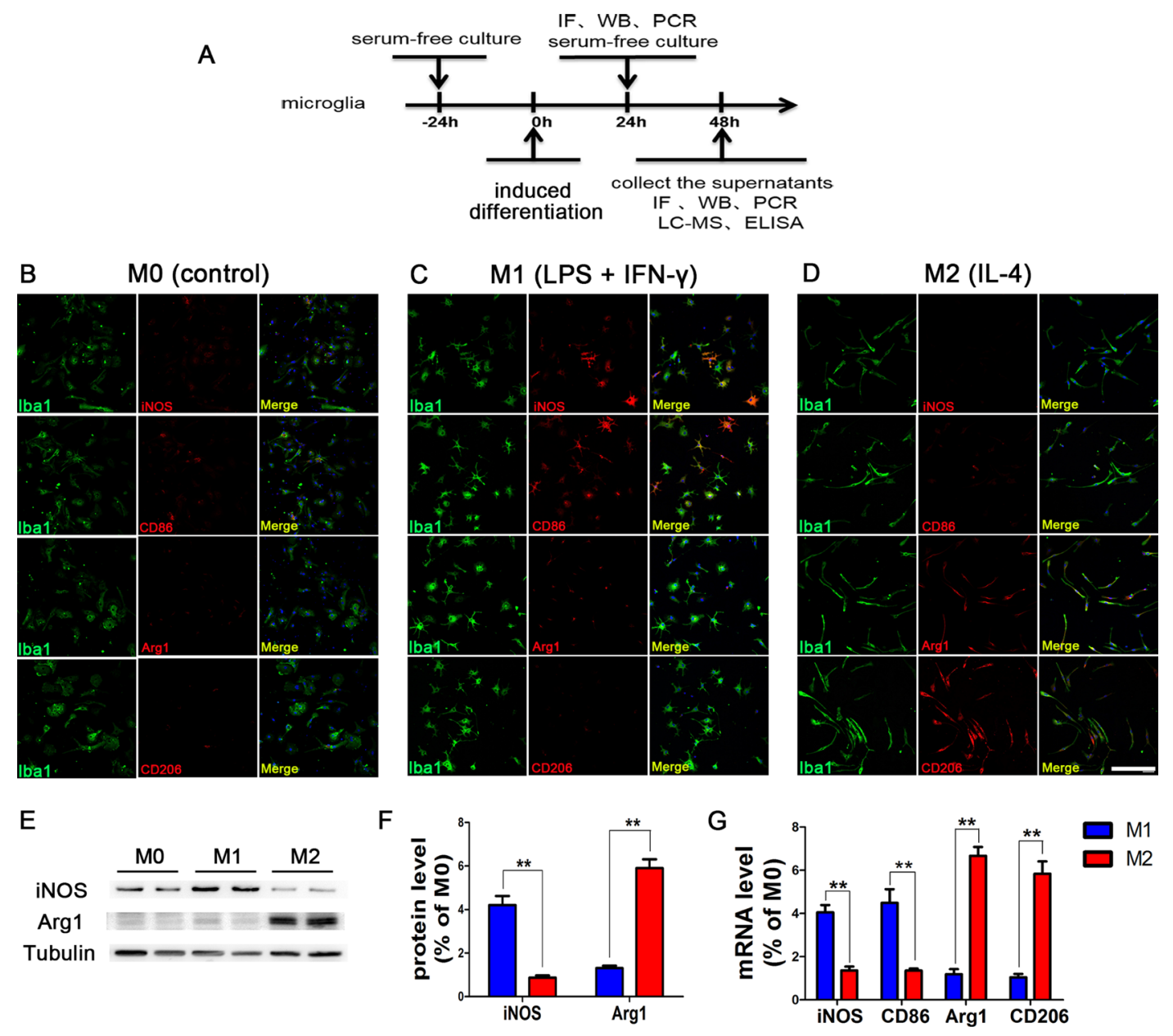

Figure 1: Phenotypic changes of polarized microglia under different intervention conditions. (A) The scheme of the experimental timeline for microglia. Phenotypic changes of polarized microglia induced by serum-free culture medium (B), LPS+IFN- $\gamma$ (C), and IL-4 (D) for $24 \mathrm{~h}$. M1 subtype-specific markers iNOS and CD86 are more highly expressed in LPS+IFN- $\gamma$ polarized microglia, while M2 subtype-specific markers CD206 and Arg1 are more highly expressed in IL-4 polarized microglia. The results of WB (E, F) and PCR (G) assays were consistent. $N=8$, bar $=50 \mu \mathrm{m}, * * P<0.01$. 


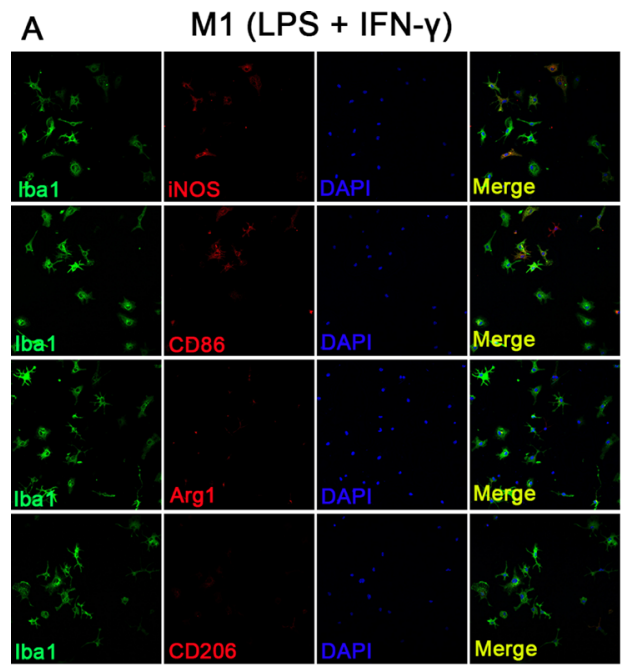

C

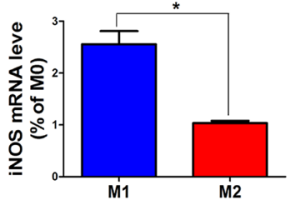

D

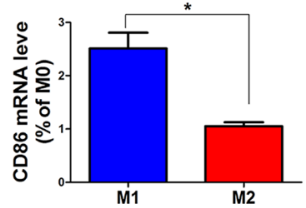

B

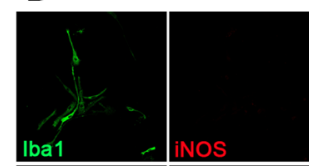
M2 (IL-4)

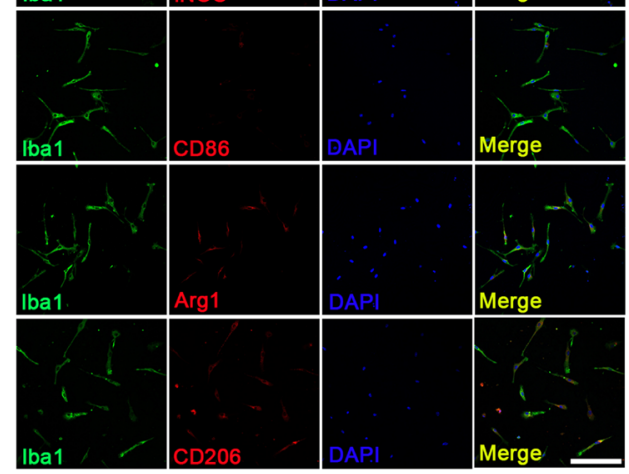

E

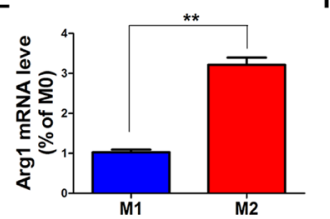

F

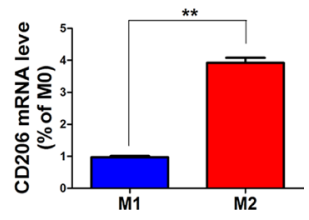

Figure 2: Phenotype maintenance of M1 and M2 microglia at $\mathbf{2 4} \mathbf{h}$ after removal of intervention. After microglia were polarized by LPS+IFN- $\gamma$ and IL-4 for $24 \mathrm{~h}, \mathrm{LPS}+\mathrm{IFN}-\gamma$ and IL-4 were replaced with serum-free culture medium, and microglia were cultured for another $24 \mathrm{~h}$. Approximately $50 \%$ of microglia polarized by LPS+IFN- $\gamma$ still expressed M1 subtype-specific markers iNOS and CD86, and iNOS mRNA and CD86 mRNA were still highly expressed (A). Approximately, about half of the microglia polarized by IL-4 expressed the M2 subtype-specific markers CD206 and Arg1 (B). The expression levels of these two markers differed from those in M0 microglia, and the differences were statistically significant $(\mathbf{C}-\mathbf{F}) . N=8$, bar $=50 \mu \mathrm{m} . * P<0.05, * * P<0.01$.
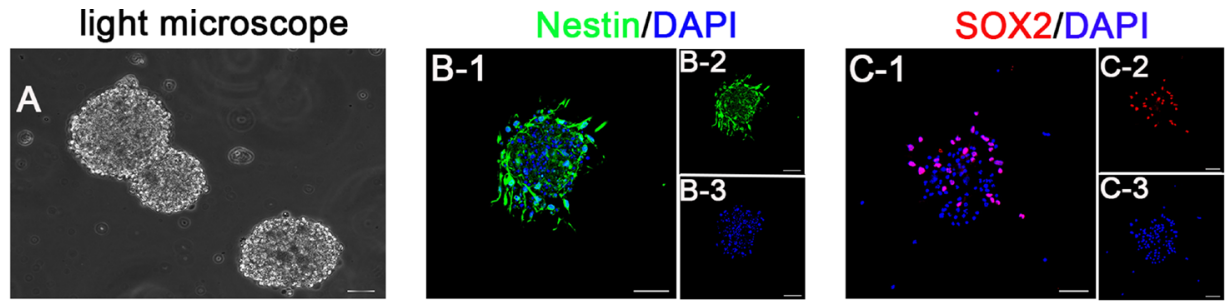

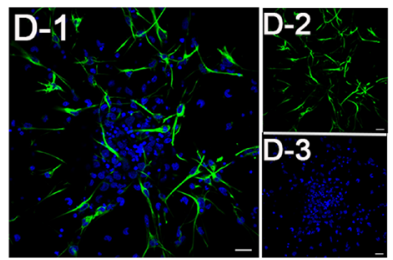

Tuj-1/DAPI

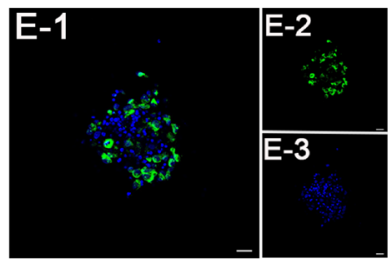

Olig2/DAPI

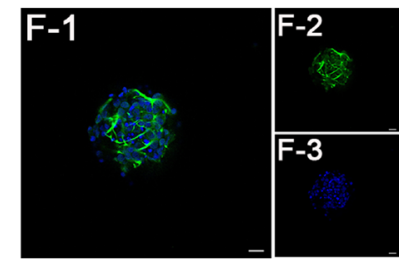

GFAPIDAPI

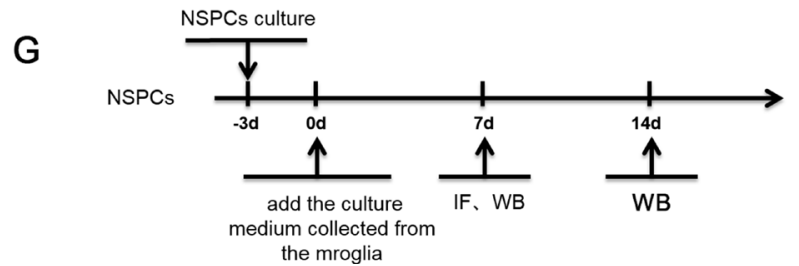

Figure 3: (A) The phase contrast photo showed the suspended growth of neurospheres. (B) and (C) The immunostaining depicted isolated cells expressing Nestin (green) and SOX2 (red). (D-F) The immunofluorescence identification for the differentiation potential of NSPCs into neurons (Tuj-1), astrocytes (GFAP), and oligodendrocytes (Olig2), respectively. Cell nuclei was stained with DAPI in blue. (G) A scheme of the experimental timeline for NSPCs. Bar $=20 \mu \mathrm{m}$. 
neurogenesis and oligodendrogenesis. With GW9662 inhibited, the percentage of GFAP + cells significantly increased. These results demonstrated that inhibition of PPAR $\gamma$ could promote NSPCs differentiation towards astrocytes, and limit the differentiation towards neurons and oligodendrocytes.

\section{DISCUSSION}

Our study discovers that, when induced by anti-inflammatory M2 microglia supernatant, NSPCs differentiated significantly more into neurons and oligodendrocytes and less into astrocytes. M2 supernatant promotes NSPCs differentiation through the secretion of $15 \mathrm{~d}_{-} \mathrm{PGJ}_{2}$, which can activate PPAR $\gamma$ in NSPCs nucle. Our research confirm that M2 microglia can promote neurogenesis and oligodendrogenesis from NSPCs differentiation through the PPAR $\gamma$ pathway.

As the resident immune cells in CNS, microglia are key mediators of secondary inflammation. M2 phenotypic microglia can improve neural repair after CNS injury [21-25]. In the present study, microglia polarized by IL-4 became long and spindle-like, the expression levels of M2 subtype markers significantly increased, and the
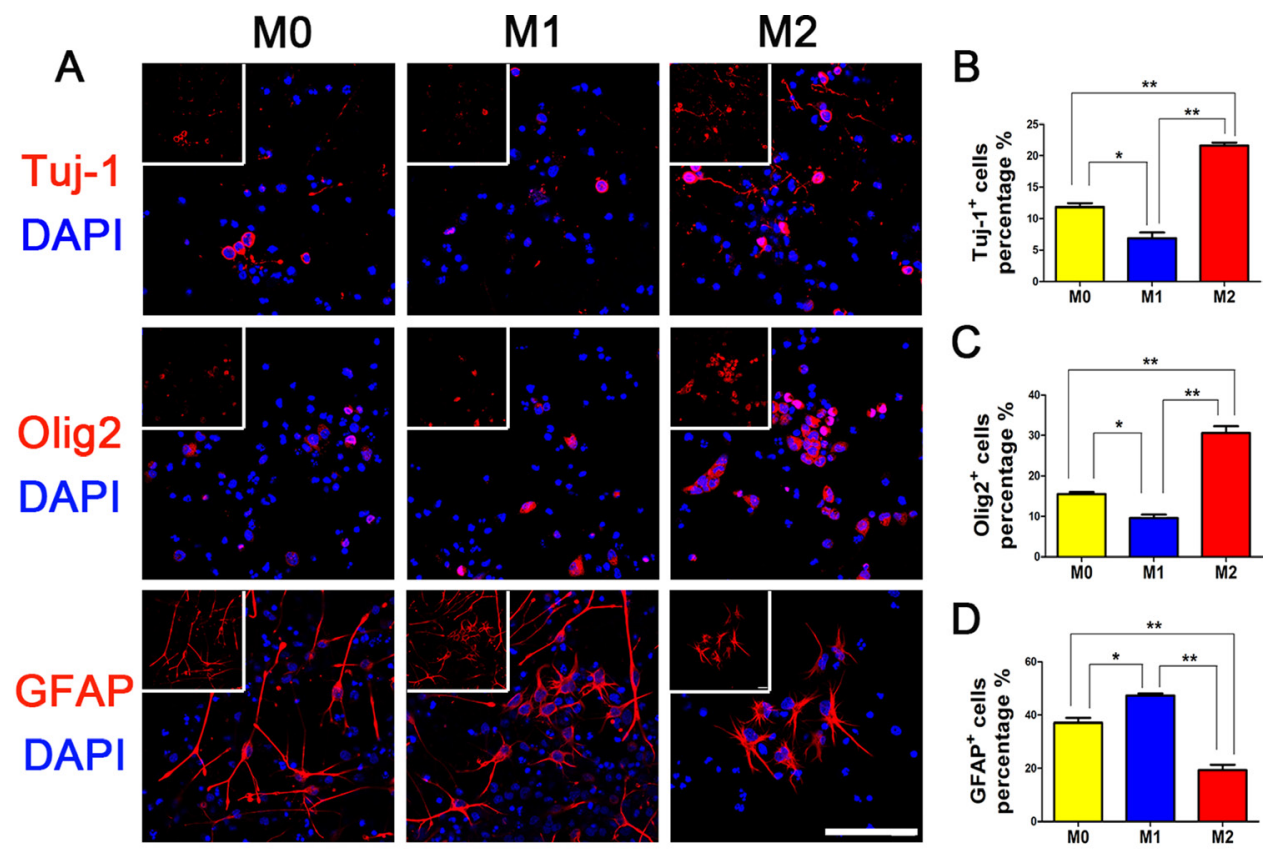

C
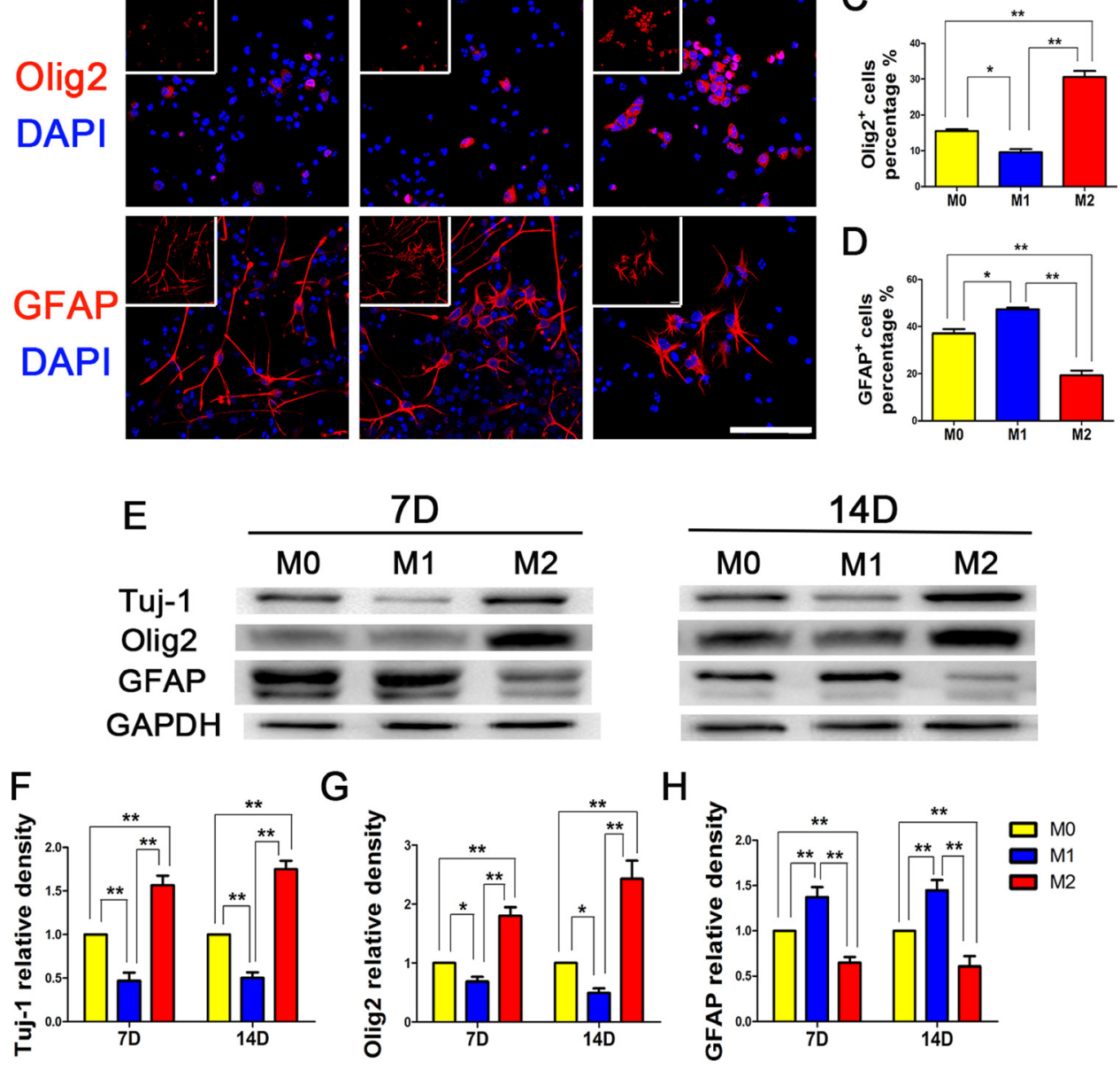

Figure 4: NSPCs differentiation induced by different microglia supernatants. M0, M1, and M2 microglia supernatants were separately collected and added to 1\% FBS to induce NSPCs differentiation for 14 days. (A) Immunofluorescence, (B-D) Statistical analysis for the differentiation percentage of neurons (Tuj-1), oligodendrocytes (Olig2) and astrocytes (GFAP) from NSPCs, respectively. WB results (E) showed that M2 microglia supernatant up-regulated Tuj-1 and Olig2 expression levels and reduced GFAP expression in NSPCs. The differences in Tuj-1, Olig2 and GFAP+ cells induced by M0, M1, and M2 microglia supernatants were all statistically significant (F-H). $N=8$, bar $=50 \mu \mathrm{m} .{ }^{*} P<0.05, * * P<0.01$. 
percentage of oligodendrocytes and neurons differentiated from NSPCs exposed to M2 microglia supernatant were increased. This result is consistent with a previous report [12]. At the same time, we also found that M1 microglia induced by LPS + IFN $-\gamma$ promoted astrocyte differentiation, while Butovsky discovered that IFN- $\gamma$ activated microglia showed a bias towards neurogenesis. We found that the discrepancy was caused by different cell models. Butovsky et al. used microglia activated by IFN- $\gamma$ and used cover slips coated with matrigel to co-culture microglia and NSPCs [12]. However, our model combined LPS+IFN- $\gamma$ to promote microglia activation; we then collected the supernatant to prepare the culture medium used to induce differentiation. When inoculating NSPCs, we avoided using dishes pretreated with any biomaterial substrates; because dishes pretreated with poly-L-ornithine could induce NSPCs differentiation towards neurons and oligodendrocytes [26]. Zhang et al. used a similar model and reached a relatively consistent conclusion [27]. The research indicated that M2 microglia could assist NSPCs migration to the injury region and promote neurogenesis.

There remains a lack of in-depth studies on how M2 microglia promotes neurogenesis and oligodendrogenesis. In this study, we found that the released $15 \mathrm{~d}_{-} \mathrm{PGJ}_{2}$ in the M2 microglia supernatant was significantly increased compared to that in the M1 supernatant. Additionally, $15 \mathrm{~d}-\mathrm{PGJ} \mathrm{J}_{2}$-rich supernatant could activate PPAR $\gamma$ in NSPCs, significantly increase the number of Tuj-1+ and Olig2+ cells generated during NSPCs differentiation, and reduce the number of GFAP+ cells. However, after intervention with the PPAR $\gamma$ inhibitor GW9662, NSPCs differentiation exhibited the opposite phenomenon. These data suggest that $15 \mathrm{~d}-\mathrm{PGJ}_{2}$ and PPAR $\gamma$ are important factors for M2 microglia promotion of NSPCs differentiation.

$15 \mathrm{D}-\mathrm{PGJ}_{2}$ is derived from $\mathrm{PGD}_{2}$, a major cyclooxygenase 2 product synthesized in a variety of tissues under inflammatory conditions [28]. As the receptor for $15 \mathrm{~d}_{-} \mathrm{PGJ}_{2}, \mathrm{PPAR}$ is a key factor in glucose homeostasis,
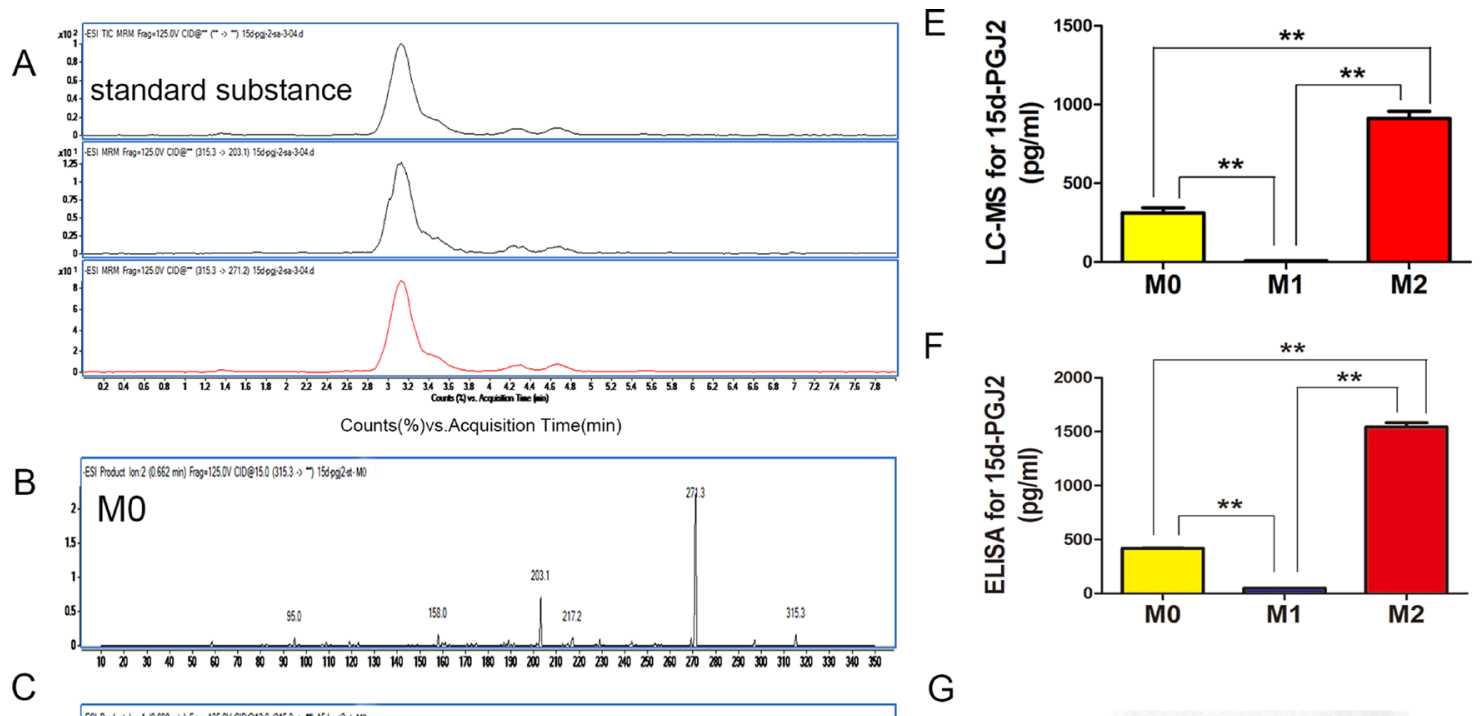

C
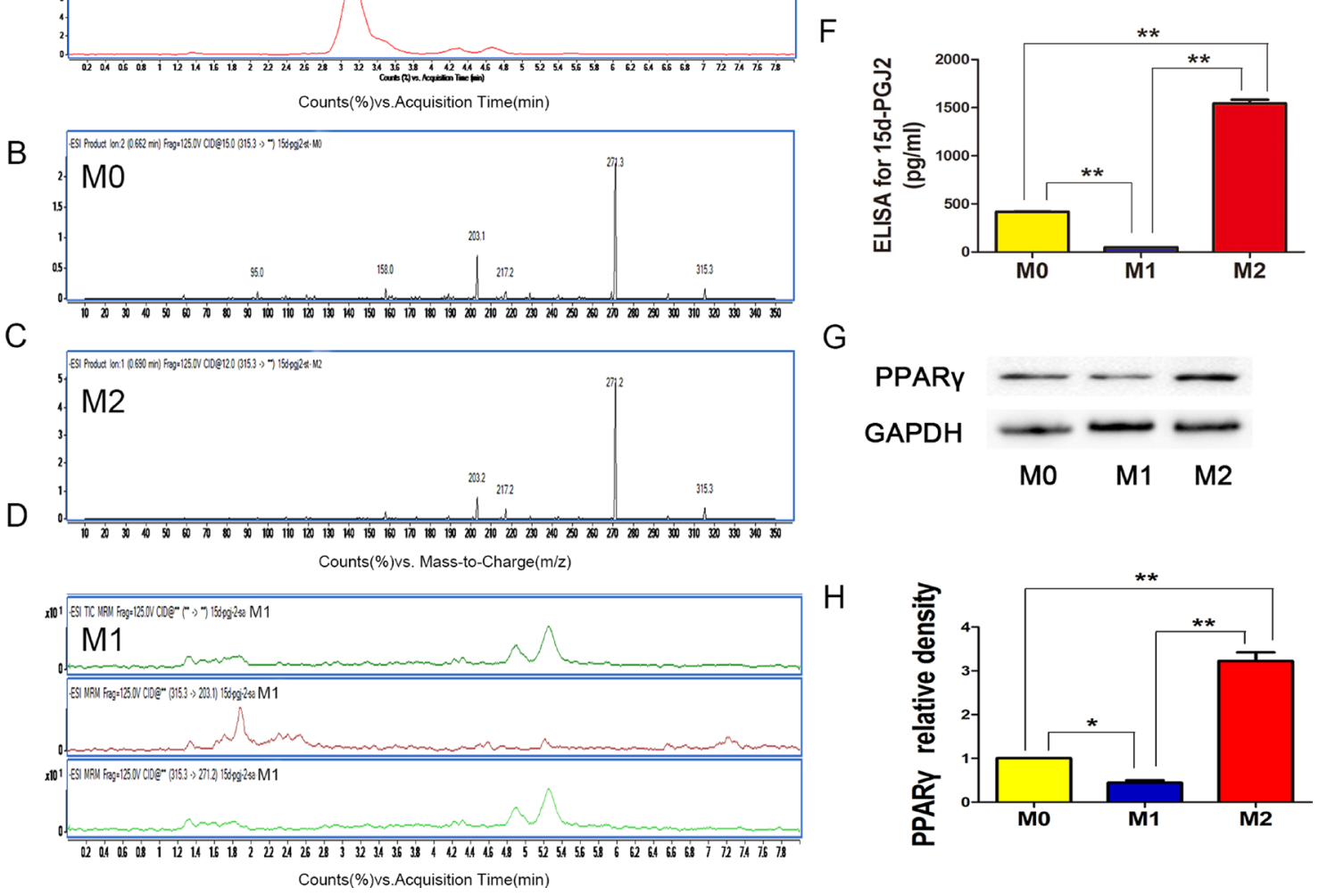

Figure 5: 15D-PGJ2 released in M0, M1, and M2 microglia. (A) With $12 \mathrm{~V}$ or $15 \mathrm{~V}$ voltage, we divided the solvent system (315.3 molecular weight, M-H-) into the quantitative ion (271.2 molecular weight) and the qualitative ion (203.1 molecular weight), respectively. (B-D) LC-MS analysis for 15d-PGJ in M0, M1, and M2 microglia. (E) Statistical analysis for level of 15d-PGJ 2 with LC-MS. (F) Statistical analysis for $15 \mathrm{~d}_{-} \mathrm{PGJ}_{2}$ level with ELISA. (G, H) WB for PPAR $\gamma$ expression in NSPCs induced with different microglia supernatants. $N=8, * P<0.05, * * P<0.01$. 
lipid metabolism and the regulation of cellular functions in a ligand-dependent manner [29-31]. PPAR $\gamma$ also plays an important role in the process of NSPCs differentiation. Morales et al. significantly increased the numbers of MAP-
2- and GFAP-positive cells by using the PPAR $\gamma$ agonist pioglitazone, and the addition of GW9662 completely abolished these effects [32]. It has also been reported that PPAR $\gamma$ agonists can increase the quantities of TuJ1-

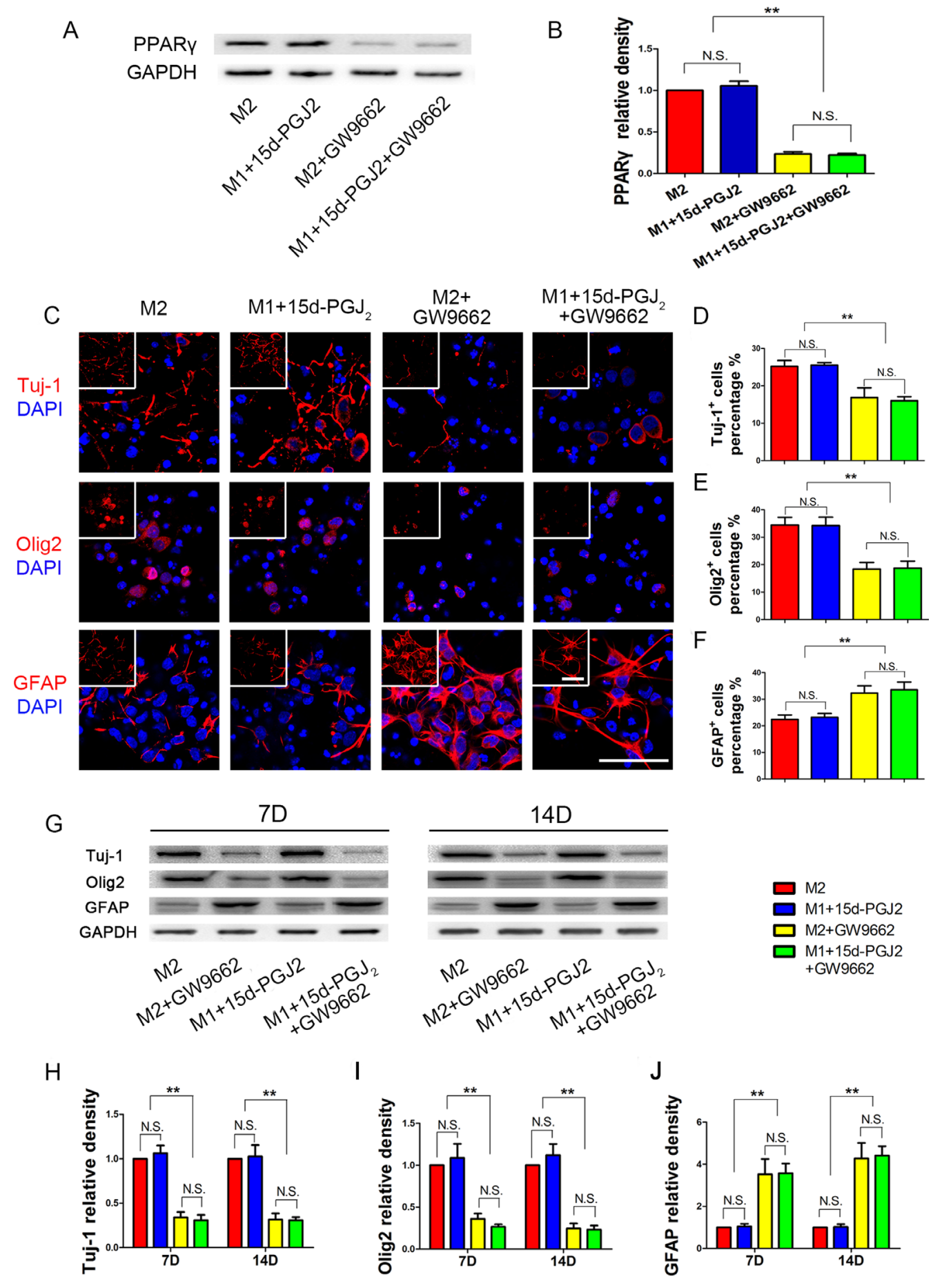

Figure 6: Inhibition of PPAR $\gamma$ reduced NSPCs differentiation induced by M2 microglia towards neurons and oligodendrocytes. GW9662 $(30 \mu \mathrm{mol} / \mathrm{ml})$ was added $1 \mathrm{~h}$ in advance to block PPAR $\gamma$ activity, and then M2 supernatant or M1 supernatant $+15 \mathrm{~d}-\mathrm{PGJ}_{2}(1 \mathrm{ngl} / \mathrm{ml}$, the same concentration with M1 supernatant) were added to induce NSPCs differentiation. During the entire differentiation process, the concentrations of $15 \mathrm{~d}_{-} \mathrm{PGJ}_{2}$ and GW9662 were maintained at $1 \mathrm{ngl} / \mathrm{ml}$ and $30 \mu \mathrm{mol} / \mathrm{ml}$, respectively. (A, B) WB showed PPAR $\gamma$ expression on day 14 during NSPCs differentiation. (C) Immunofluorescence, (D-F) Statistical analysis for neurons (Tuj-1), oligodendrocytes (Olig2) and astrocytes (GFAP) differentiation percentage from NSPCs, respectively. (G) WB results. (H-J) Statistical analysis of the WB. $N=8$, bar $=50 \mu \mathrm{m}$. N.S. indicates no significant difference, $* P<0.05, * * P<0.01$. 
and CNPase-positive cells, while the PPAR $\gamma$-selective antagonist GW9662 can reduce quantities of TuJ1- and CNPase-positive cells [33, 34]. Further, it has been reported that PPAR $\gamma$ can promote NSPCs differentiation towards astrocytes [35]. The results of our experiments indicate that M2 microglia can activate PPAR $\gamma$ expression in NSPCs and promote neurogenesis. Additionally, these data demonstrate that PPAR $\gamma$ plays an important role in NSPCs differentiation.

In summary, the present study illustrates that M2 microglia supernatant can increase the percentage of neurons and oligodendrocytes generated by NSPCs differentiation, and decrease the number of differentiated astrocytes. M2 microglia promotes NSPCs differentiation by activating the PPAR $\gamma$ signaling pathway. Our experiment provides further experimental support and alternative options for promoting NSPCs-oriented differentiation.

\section{MATERIALS AND METHODS}

\section{Animal}

This study was performed in accordance with the China's animal welfare legislation for the care and use of animals and approved by the Third Military Medical University in Chongqing, China. We did our best to minimize the number of animals and decrease their suffering. E14.5 ICR mice were sacrificed after anesthetized with pentobarbital (60 mg/kg intraperitoneally).

\section{Microglia culture}

The cortex of newborn mice was dissected and the cerebral pia mater was stripped under an anatomical microscope. The tissue was digested in $0.125 \%$ trypsin for $8 \mathrm{~min}$ and stopped from digestion with $10 \%$ fetal bovine serum (FBS). Then, the digested material was filtered with 200-mesh metal strainer. After centrifugation of the filtrate, a cell suspension was prepared in DMEM/F12 containing $1 \%$ double antibodies and 10\% FBS, placed into a $5 \% \mathrm{CO}_{2}$ incubator and incubated at $37^{\circ} \mathrm{C}$ for $12 \mathrm{~h}$; the suspension was replaced after the cells adhered to the walls. Thereafter, the culture medium was replaced once every three days. On day 14, the culture was placed on a shaker at $250 \mathrm{rpm}$ for $1 \mathrm{~h}$, the supernatant was collected, and microglia were obtained. As identified by Ibal staining, the purity of the microglia preparation was greater than $95 \%$.

\section{Polarization of microglia and preparation of NSPCs conditional culture media}

Purified microglia were spread on a six-well plate at the concentration of $10^{6} /$ well, serum deprived, and cultured for $24 \mathrm{~h}$. Then, serum-free culture medium was used to induce M0 microglia, serum-free culture medium containing lipopolysaccharides (LPS, $100 \mathrm{ng} / \mathrm{ml})+$ IFN- $\gamma$ $(25 \mu \mathrm{g} / \mathrm{ml})$ was used to induce M1 microglia, and serumfree culture medium containing IL-4 $(20 \mu \mathrm{g} / \mathrm{ml})$ was used to induce M2 microglia. The microglia were cultured under the three culture medium conditions for $24 \mathrm{~h}$, after which the media was replaced, and then serum-free culture media was added to continue culturing. After $24 \mathrm{~h}, 1 \%$ FBS was added to the collected the supernatants, and the culture media was used to induce NSPC differentiation.

\section{NSPCs culture}

The mouse embryos were obtained from the 14.5-day pregnant mice. The cortex was dissected and cut into pieces, digested in $0.125 \%$ trypsin for $8 \mathrm{~min}$, stopped from digestion with soybean trypsin inhibitor, and filtered with a $100 \mu \mathrm{m}$ Nylon cell strainer. The cells were re-suspended in NSPC culture medium at an initial concentration of $1 * 10^{5}$ cells $/ \mathrm{mL}$, and cultured in a $5 \%$ $\mathrm{CO}_{2}$ incubator at $37^{\circ} \mathrm{C}$. The NSPC culture medium was DMEM/F12 complete medium containing $20 \mathrm{ng} / \mathrm{ml}$ recombinant murine FGF (PeproTech, 450-33), 20 ng/ml recombinant murine EGF (PeproTech, 315-09), and 2\% B-27 supplement (Life Technologies, 17504-044). After culture for three days, the culture was centrifuged at $800 \mathrm{rpm}$ for $5 \mathrm{~min}$, and then the cells were re-suspended in culture medium for induced NSPC differentiation. 15D-PGJ ${ }_{2}$ (Sigma, D8440, $1 \mathrm{ng} / \mathrm{ml}$ ) and GW9662 (Sigma, $\mathrm{M} 6191,30 \mu \mathrm{mol} / \mathrm{ml}$ ) were added to the conditional culture media. Half of the volume of culture medium for induced differentiation was replaced every three days.

\section{Apoptosis assay}

Annexin-V staining was used to detect the death/ survival of NSPCs with an Annexin V-FITC Apoptosis Detection Kit (Beyotime) and processed in accordance with the manufacturer recommendation. $2 * 10^{6}$ cells were cultured with NSPC culture medium for 3 days. Then supernatants collected from M0, M1, M2 microglia were added to induce NSPC differentiation. On day 3, 7 and 14, adherent cells were collected with StemPro ${ }^{R}$ Accutase ${ }^{\mathrm{R}}$ Cell Dissociation Reagent (Life Technologies, A1110501). Cells were incubated with Annexin V-FITC at room temperature for 10 minutes and re-suspended with binding buffer. Then Propidium Iodide was added into suspension for 5 minutes on ice. Cells were analyzed using a FACScan flow cytometer.

\section{lactate dehydrogenase (LDH) release assays}

Cell necrosis were measured with lactate dehydrogenase $(\mathrm{LDH})$ release assays. $100 \mu \mathrm{l}$ of cell suspension $(\sim 10000$ cells/well) was dispensed in a 96well cell culture cluster with NSPC culture medium for 3 days. Then NSPCs differentiation was induced with different phenotypes microglia supernatants. Supernatants 
were collected on day 3, 7 and 14. Then cells were lysed with $2 \%$ Triton X-100 for 15 min to release all LDH. The cell lysis were used as positive controls to determine the maximal LDH release. $\mathrm{LDH}$ release was detected using the absorbance of the culture medium at a test wavelength of $450 \mathrm{~nm}$ with a microplate reader according to the manufacturer's instructions.

\section{Cell proliferation assay}

Cell counting Kit- 8 was used to assess cell proliferation. $10^{4}$ cells/well NSPCs were cultured in a 96well plate and induced differentiation with M0, M1, M2 supernatants. On day 3, 7 and 14, the cells were incubated with $10 \%(\mathrm{v} / \mathrm{v})$ WST solution for $2.5 \mathrm{~h}$ at $37^{\circ} \mathrm{C}$. Then, the absorbance of the culture medium at a test wavelength of $450 \mathrm{~nm}$ was determined using a microplate reader and a reference wavelength of $630 \mathrm{~nm}$ as well.

\section{Immunofluorescence}

Intervened cells were fixed in 4\% paraformaldehyde, penetrated with $0.1 \%$ Triton $\mathrm{X}-100$, blocked with $5 \%$ bovine serum albumin (BSA) and incubated with primary antibodies at $4{ }^{\circ} \mathrm{C}$ overnight. Afterwards, the cells were stained with fluorescein-labeled secondary antibodies and 4',6-diamidino-2-phenylindole dihydrochloride (DAPI) under dark conditions, sealed on the slides, and observed under a laser confocal microscope. The respective concentrations of primary antibodies were: Iba1 (wako, Lot: 019-19741, 1:1000), CD86 (BD, Lot: 553689, 1:200), iNOS (BD, Lot: 610328, 1:200), CD206 (Santa, sc-34577, 1:100), Arg1 (Santa, sc-18351, 1:100), Nestin (Abcam, ab6142, 1:200), SOX2 (Abcam, ab97597, 1:200), Tuj1 (Abcam, ab78078, 1:200), Olig2 (Abcam, ab109186, 1:100), GFAP (Abcam, ab7260, 1:1000), MAP2 (Abcam, ab5392, 1:500), O4 (Sigma, O7139, 1:100), A minimum of four images was captured using a $20 \mathrm{X}$ objective on a Zeiss microscope (Zeiss AxioCam, Germany) and the positive cells were counted using Image J.

\section{Western blot (WB)}

Total protein was extracted with radioimmunoprecipitation assay (RIPA) lysis buffer. A volume containing $20 \mu \mathrm{g}$ protein was loaded onto a $10 \%$ sodium dodecyl sulfate polyacrylamide gel electrophoresis (SDS-PAGE) gel, electrophoresed and transferred to polyvinylidene difluoride (PVDF) membrane. Then the membrane was blocked with 5\% BSA, incubated with iNOS (BD, 1:4000), Arg1 (Santa, 1:500), Tuj-1 (Abcam, 1:1000), Olig2 (Abcam, 1:2000), GFAP (Abcam, ab 7260, 1:10000), PPAR $\gamma$ (Santa, sc-7196, 1:1000), or $\beta$-catenin (Abcam, ab 32572, 1:10000) primary antibody at $4^{\circ} \mathrm{C}$ overnight, and then incubated with secondary antibodies at room temperature for $2 \mathrm{~h}$. Images were developed using a gel imaging system.

\section{Real-time polymerase chain reaction (RT-PCR)}

The primer was designed and synthesized by Shanghai Sangon Biological Engineering Technology \& Services Company. Total ribonucleic acid (RNA) was extracted using the TRIzol method, and the concentration and purity were detected using the TaKaRa quantitative PCR kit according to the reagent instructions. The reverse transcription and amplification system was performed under the reverse transcription conditions of $42^{\circ} \mathrm{C}$ for $15 \mathrm{~min}$ and $85^{\circ} \mathrm{C}$ for $5 \mathrm{sec}$ and the amplification conditions of $95^{\circ} \mathrm{C}$ for $30 \mathrm{sec}$ followed by 40 cycles of $95^{\circ} \mathrm{C}$ for $5 \mathrm{sec}$ and $60^{\circ} \mathrm{C}$ for $30 \mathrm{sec}$; the resulting images were developed using a gel imaging system. The related primers were designed as follows:

\begin{tabular}{|l|l|l|}
\hline & forward primer & reverse prime \\
\hline iNOS & $\begin{array}{l}\text { GGCACAGGGTCATCA } \\
\text { TCAAA }\end{array}$ & $\begin{array}{l}\text { TCAGGTCACTTTGG } \\
\text { TAGGATTT }\end{array}$ \\
\hline CD86 & $\begin{array}{l}\text { GTAGAGTCCAGTTGTT } \\
\text { CCTGTC }\end{array}$ & $\begin{array}{l}\text { TGGTTCTGTACGAG } \\
\text { CACTATTT }\end{array}$ \\
\hline arginase-1 & GTCCCTAATGACAGCT & $\begin{array}{l}\text { CCACACTGACTCTT } \\
\text { CCATTCTT }\end{array}$ \\
\hline CD206 & $\begin{array}{l}\text { GTGGTCCTCCTGATTG } \\
\text { TGATAG }\end{array}$ & $\begin{array}{l}\text { CACTTGTTCCTGGA } \\
\text { CTCAGATTA }\end{array}$ \\
\hline
\end{tabular}

\section{ELISA}

Microglia under the three culture medium conditions were cultured for $24 \mathrm{~h}$, then the media was replaced, and serum-free culture media was added to continue culturing. After $24 \mathrm{~h}$, supernatants were collected from each group. The supernatants were collected and evaluated in duplicate using $15 \mathrm{~d}_{-} \mathrm{PGJ}_{2}$ ELISA kits in accordance with the manufacturer's guidelines. After preparing the standard samples, those to be tested were then incubated for $30 \mathrm{~min}$ at $37^{\circ} \mathrm{C}$. After repeated washings in PBS, the ELISA reagents were added, followed by a $30-\mathrm{min}$ incubation at $37^{\circ} \mathrm{C}$. Finally, the developing solution was added, and the absorbance of each well was measured at $450 \mathrm{~nm}$ in a microplate reader. The average absorbance values for each set of standards and samples were calculated, and a standard curve was constructed. The concentrations of the samples were calculated from the standard curve.

\section{LC-MS}

Different phenotypes of microglia supernatants were acidified with $\mathrm{HCl}(1 \mathrm{~N})$ to $\mathrm{pH} 3.0$ and extracted with ethyl acetate. With $12 \mathrm{v}$ or $15 \mathrm{v}$ voltage, we divided

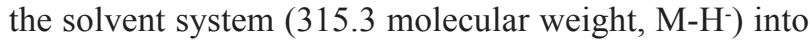
the quantitative ion (271.2 molecular weight) and the qualitative ion (203.1 molecular weight), respectively. LC-MS/MS system (Thermo Fisher Agilent, 641013) was used to abtain the data. As an internal control to calculate extraction efficiency, $200 \mathrm{ng}$ of deuterated $15 \mathrm{~d}_{-} \mathrm{PGJ}_{2}$ (d4) (sigma) was used, which showed a 70\% extraction efficiency. All experiments were performed by the Third 
Military Medical University Biomedical Analysis and Test Center.

\section{Data analysis}

All data were presented as the mean \pm standard error. SPSS16.0 software (SPSS Inc, Chicago, IL) was used for statistical analysis. One-way ANOVA with the appropriate Tukey's post hoc post hoc test was used to compare experimental groups. ${ }^{*} P<0.05$ was considered statistically significant.

\section{CONFLICTS OF INTEREST}

The authors declare no competing financial interests.

\section{GRANT SUPPORT}

This work was supported by grants from National Natural Science Foundation of China (No. 81571214 and No. 81371440), and Southwest Hospital Science Foundation (SWH2016JSYB-03).

\section{Authors' contributions}

This study was based on the original idea of JKL and JCY. JCY and HFG carried out the immunofluorescence assay and drafted the manuscript. HTZ, YY and XZ performed data analyses. WL, YY, YXC and WJW carried out the molecular biology studies, YFY and WXC provided illustrations. JKL and JCY were responsible for supervising all experiments, data analyses and the drafting of the manuscript. All authors read and approved the final manuscript.

\section{REFERENCES}

1. Chu W, Yuan J, Huang L, Xiang X, Zhu H, Chen F, Chen Y, Lin J, Feng H. Valproic Acid Arrests Proliferation but Promotes Neuronal Differentiation of Adult Spinal NSPCs from SCI Rats. Neurochem Res. 2015; 40:1472-1486.

2. Iwasaki M, Wilcox JT, Nishimura Y, Zweckberger K, Suzuki H, Wang J, Liu Y, Karadimas SK, Fehlings MG. Synergistic effects of self-assembling peptide and neural stem/progenitor cells to promote tissue repair and forelimb functional recovery in cervical spinal cord injury. Biomaterials. 2014; 35:2617-2629.

3. Klose J, Schmidt NO, Melms A, Dohi M, Miyazaki J, Bischof F, Greve B. Suppression of experimental autoimmune encephalomyelitis by interleukin-10 transduced neural stem/progenitor cells. J Neuroinflammation. 2013; 10:117.

4. Nishimura S, Yasuda A, Iwai H, Takano M, Kobayashi Y, Nori S, Tsuji O, Fujiyoshi K, Ebise H, Toyama Y,
Okano H, Nakamura M. Time-dependent changes in the microenvironment of injured spinal cord affects the therapeutic potential of neural stem cell transplantation for spinal cord injury. Mol Brain. 2013; 6:3.

5. Kizil C, Kyritsis N, Brand M. Effects of inflammation on stem cells: together they strive? EMBO Rep. 2015; 16:416-426.

6. Delaby C, Pilard N, Goncalves AS, Beaumont C, CanonneHergaux F. Presence of the iron exporter ferroportin at the plasma membrane of macrophages is enhanced by iron loading and down-regulated by hepcidin. Blood. 2005; 106:3979-3984.

7. Gensel JC, Nakamura S, Guan Z, van Rooijen N, Ankeny DP, Popovich PG. Macrophages promote axon regeneration with concurrent neurotoxicity. J Neurosci. 2009; 29:3956-3968.

8. David S, Kroner A. Repertoire of microglial and macrophage responses after spinal cord injury. Nat Rev Neurosci. 2011; 12:388-399.

9. David S, Zarruk JG, Ghasemlou N. Inflammatory pathways in spinal cord injury. Int Rev Neurobiol. 2012; 106:127-152.

10. Gordon S, Taylor PR. Monocyte and macrophage heterogeneity. Nat Rev Immunol. 2005; 5:953-964.

11. Satoh T, Kidoya H, Naito H, Yamamoto M, Takemura N, Nakagawa K, Yoshioka Y, Morii E, Takakura N, Takeuchi O, Akira S. Critical role of Trib1 in differentiation of tissueresident M2-like macrophages. Nature. 2013; 495:524-528.

12. Butovsky O, Ziv Y, Schwartz A, Landa G, Talpalar AE, Pluchino S, Martino G, Schwartz M. Microglia activated by IL-4 or IFN-gamma differentially induce neurogenesis and oligodendrogenesis from adult stem/progenitor cells. Mol Cell Neurosci. 2006; 31:149-160.

13. Forman BM, Tontonoz P, Chen J, Brun RP, Spiegelman BM, Evans RM. 15-Deoxy-delta 12, 14-prostaglandin J2 is a ligand for the adipocyte determination factor PPAR gamma. Cell. 1995; 83:803-812.

14. Huang L, Li G, Feng X, Wang L. 15d-PGJ2 Reduced Microglia Activation and Alleviated Neurological Deficit of Ischemic Reperfusion in Diabetic Rat Model. Biomed Res Int. 2015; 2015:864509.

15. Kim SE, Lee EO, Yang JH, Kang JH, Suh YH, Chong YH. 15-deoxy-Delta, -prostaglandin $\mathrm{J}$ inhibits human immunodeficiency virus-1 tat-induced monocyte chemoattractant protein-1/CCL2 production by blocking the extracellular signal-regulated kinase-1/2 signaling pathway independently of peroxisome proliferator-activated receptorgamma and heme oxygenase-1 in rat hippocampal slices. J Neurosci Res. 2012; 90:1732-1742.

16. Zhang F, Liu F, Yan M, Ji H, Hu L, Li X, Qian J, He X, Zhang L, Shen A, Cheng C. Peroxisome proliferatoractivated receptor-gamma agonists suppress iNOS expression induced by LPS in rat primary Schwann cells. J Neuroimmunol. 2010; 218:36-47.

17. Nelson SM, Shay AE, James JL, Carlson BA, Urban JF, Jr. Prabhu KS. Selenoprotein Expression in Macrophages 
Is Critical for Optimal Clearance of Parasitic Helminth Nippostrongylus brasiliensis. J Biol Chem. 2016; 291:2787-2798.

18. Gandhi UH, Kaushal N, Ravindra KC, Hegde S, Nelson SM, Narayan V, Vunta H, Paulson RF, Prabhu KS. Selenoproteindependent up-regulation of hematopoietic prostaglandin D2 synthase in macrophages is mediated through the activation of peroxisome proliferator-activated receptor (PPAR) gamma. J Biol Chem. 2011; 286:27471-27482.

19. Vunta H, Davis F, Palempalli UD, Bhat D, Arner RJ, Thompson JT, Peterson DG, Reddy CC, Prabhu KS. The anti-inflammatory effects of selenium are mediated through 15-deoxy-Delta12,14-prostaglandin J2 in macrophages. J Biol Chem. 2007; 282:17964-17973.

20. Katura T, Moriya T, Nakahata N. 15-Deoxy-delta 12,14-prostaglandin $\mathrm{J} 2$ biphasically regulates the proliferation of mouse hippocampal neural progenitor cells by modulating the redox state. Mol Pharmacol. 2010; 77:601-611.

21. Liu J, Hjorth E, Zhu M, Calzarossa C, Samuelsson EB, Schultzberg M, Akesson E. Interplay between human microglia and neural stem/progenitor cells in an allogeneic co-culture model. J Cell Mol Med. 2013; 17:1434-1443.

22. Sakaguchi S, Shono J, Suzuki T, Sawano S, Anderson JE, Do MK, Ohtsubo H, Mizunoya W, Sato Y, Nakamura M, Furuse M, Yamada K, Ikeuchi Y, et al. Implication of anti-inflammatory macrophages in regenerative motoneuritogenesis: promotion of myoblast migration and neural chemorepellent semaphorin 3A expression in injured muscle. Int J Biochem Cell Biol. 2014; 54:272-285.

23. Matsubara K, Matsushita Y, Sakai K, Kano F, Kondo M, Noda M, Hashimoto N, Imagama S, Ishiguro $\mathrm{N}$, Suzumura A, Ueda M, Furukawa K, Yamamoto A. Secreted ectodomain of sialic acid-binding Ig-like lectin-9 and monocyte chemoattractant protein-1 promote recovery after rat spinal cord injury by altering macrophage polarity. J Neurosci. 2015; 35:2452-2464.

24. Kroner A, Greenhalgh AD, Zarruk JG, Passos Dos Santos R, Gaestel M, David S. TNF and increased intracellular iron alter macrophage polarization to a detrimental M1 phenotype in the injured spinal cord. Neuron. 2014; 83:1098-1116.

25. Kondo T, Matsuoka AJ, Shimomura A, Koehler KR, Chan RJ, Miller JM, Srour EF, Hashino E. Wnt signaling promotes neuronal differentiation from mesenchymal stem cells through activation of Tlx3. Stem cells. 2011; 29:836-846.
26. Ge H, Tan L, Wu P, Yin Y, Liu X, Meng H, Cui G, Wu N, Lin J, Hu R, Feng H. Poly-L-ornithine promotes preferred differentiation of neural stem/progenitor cells via ERK signalling pathway. Sci Rep. 2015; 5:15535.

27. Zhang K, Zheng J, Bian G, Liu L, Xue Q, Liu F, Yu C, Zhang H, Song B, Chung SK, Ju G, Wang J. Polarized Macrophages Have Distinct Roles in the Differentiation and Migration of Embryonic Spinal-cord-derived Neural Stem Cells After Grafting to Injured Sites of Spinal Cord. Mol Ther. 2015; 23:1077-1091.

28. Funk CD. Prostaglandins and leukotrienes: advances in eicosanoid biology. Science. 2001; 294:1871-1875.

29. Tontonoz P, Hu E, Spiegelman BM. Stimulation of adipogenesis in fibroblasts by PPAR gamma 2, a lipidactivated transcription factor. Cell. 1994; 79:1147-1156.

30. Szeles L, Torocsik D, Nagy L. PPARgamma in immunity and inflammation: cell types and diseases. Biochim Biophys Acta. 2007; 1771:1014-1030.

31. Michalik L, Auwerx J, Berger JP, Chatterjee VK, Glass CK, Gonzalez FJ, Grimaldi PA, Kadowaki T, Lazar MA, O’Rahilly S, Palmer CN, Plutzky J, Reddy JK, et al. International Union of Pharmacology. LXI. Peroxisome proliferator-activated receptors. Pharmacol Rev. 2006; 58:726-741.

32. Morales-Garcia JA, Luna-Medina R, Alfaro-Cervello C, Cortes-Canteli M, Santos A, Garcia-Verdugo JM, PerezCastillo A. Peroxisome prolifeaq1r-activated receptor gamma ligands regulate neural stem cell proliferation and differentiation in vitro and in vivo. Glia. 2011; 59:293-307.

33. Kanakasabai S, Pestereva E, Chearwae W, Gupta SK, Ansari S, Bright JJ. PPARgamma agonists promote oligodendrocyte differentiation of neural stem cells by modulating stemness and differentiation genes. PloS one. 2012; 7:e50500.

34. Wan Ibrahim WN, Tofighi R, Onishchenko N, Rebellato P, Bose R, Uhlen P, Ceccatelli S. Perfluorooctane sulfonate induces neuronal and oligodendrocytic differentiation in neural stem cells and alters the expression of PPARgamma in vitro and in vivo. Toxicol Appl Pharmacol. 2013; 269:51-60.

35. Cimini A, Cristiano L, Benedetti E, D'Angelo B, Ceru MP. PPARs Expression in Adult Mouse Neural Stem Cells: Modulation of PPARs during Astroglial Differentiaton of NSC. PPAR Res. 2007; 2007:48242. 